\title{
Intermittent hypoxia simulating obstructive sleep apnea causes pulmonary inflammation and activates the Nrf2/HO-1 pathway
}

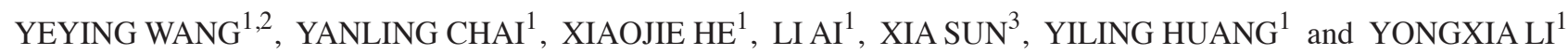 \\ ${ }^{1}$ Department of Respiratory Medicine, The Second Affiliated Hospital of Kunming Medical University, Kunming, \\ Yunnan 650101; ${ }^{2}$ Department of Epidemiology and Biostatistics, School of Public Health, Kunming Medical University, \\ Kunming, Yunnan 650500; ${ }^{3}$ Department of Infectious Diseases, Nongken Sanya Hospital, Sanya, Hainan 572000, P.R. China
}

Received September 23, 2016; Accepted May 11, 2017

DOI: $10.3892 / \mathrm{etm} .2017 .4971$

\begin{abstract}
Obstructive sleep apnea (OSA) is a disorder with high morbidity in adults. OSA damages multiple organs and tissues, including the cardiovascular and cerebrovascular systems, the metabolism system, the lungs, liver and heart. OSA-induced damage is earliest and greatest to the pulmonary tissue. The present study established a rat OSA model of differing severity by inducing intermittent hypoxia with different concentrations of $\mathrm{O}_{2}$ and it was determined that OSA caused a severe oxidative stress response and pulmonary inflammation in a dose-dependent manner. OSA increased serum levels of C-reactive protein and 8-isoprostane and elevated the expression of malondialdehyde, tumor necrosis factor $\alpha$, interleukin (IL)-1 $\beta$ and IL-6 in the pulmonary tissue. Furthermore, the expression of two important antioxidants, superoxide dismutase and glutathione, was downregulated following intermittent hypoxia. By contrast, levels of cylooxygenase 2 and inducible nitric oxide synthase, which are crucial in the antioxidative response, increased. In addition, OSA activates the nuclear factor erythroid 2-related factor 2 (Nrf2)/heme oxygenase (OH)-1 antioxidative signaling pathway. Finally, all increases and decreases in levels of inflammatory and antioxidative substances were dependent on oxygen concentrations. Therefore, the present study demonstrated that OSA, simulated by intermittent hypoxia, caused an oxidative stress response and pulmonary inflammation, and activated the canonical antioxidative Nrf2/HO-1 signaling pathway in a dose-dependent manner. These results may facilitate the development of clinical therapies to treat pulmonary diseases caused by OSA.
\end{abstract}

Correspondence to: Dr Yongxia Li, Department of Respiratory Medicine, The Second Affiliated Hospital of Kunming Medical University, 374 Dianmian Avenue, Kunming, Yunnan 650101, P.R. China

E-mail: yxl_totoro@163.com

Key words: intermittent hypoxia, obstructive sleep apnea, oxidative stress, inflammation, nuclear factor erythroid 2-related factor 2

\section{Introduction}

Sleep apnea is a disorder characterized by repetitive pauses in breathing during sleep. There are two types of sleep apnea differing in etiology: Central sleep apnea (CSA) caused by altered control of breathing and obstructive sleep apnea (OSA) caused by airway occlusion (1). OSA is the most common type, with an incidence rate 22\% (range, 9-37\%) in males and $17 \%$ (range, $4-50 \%$ ) in females (2). Airway occlusion is caused by adenoid hypertrophy, nasopharyngeal tumor, obesity or aged muscle relaxation. This manifests as snoring and apnea during sleep leading to intermittent hypoxia, which causes a hypoxic response in various tissues and organs $(3,4)$.

Various studies have investigated the effect of OSA on the cardiovascular and cerebrovascular systems. OSA has been suggested as a potential risk factor for stroke and studies conducted in the 1990s reported that the prevalence of OSA among patients with stroke was $>60 \%$ (4-6). OSA is associated with hypertension and one study demonstrated that $20 \%$ of hypertensive patients also had OSA (7). Furthermore, patients with severe OSA, defined by an apnea-hypopnea index (AHI) $\geq 30$, experience more frequent atrial fibrillation, non-sustained ventricular tachycardia and ventricular ectopy than patients with an AHI <5 (8). Men aged 40-70 years old with an AHI $\geq 30$ are $68 \%$ more likely to develop coronary heart disease and experience myocardial infarction, revascularization and cardiac death than those with an AHI $\leq 5$ (9). It has also been demonstrated that OSA causes substance and energy metabolism disorders, leading to obesity, diabetes, dyslipidemia and hyperuricemia, which all increase the risk of cardiovascular and cerebrovascular diseases (10).

OSA induces tissue damage, particularly in the pulmonary tissue. Intermittent hypoxia (IH) may cause pulmonary inflammation, leading to pulmonary fibrosis (11). A recent study found that among 92 newly diagnosed patients with idiopathic pulmonary fibrosis, 55 had an $\mathrm{AHI} \geq 15$ (12). It has also been reported that IH causes pulmonary artery vasoconstriction and pulmonary hypertension, leading to right ventricular overload and left ventricular dysfunction (13). Additionally, IH may induce inflammation in the liver and heart, aggravate inflammation in the lung and promote metastasis of melanoma to the lung (4-15). 
The aim of the present study was to induce a model of OSA in rats via $\mathrm{IH}$ and determine the mechanism of pulmonary inflammation caused by OSA.

\section{Materials and methods}

Animal model. A total of 30 healthy 8-week old male Wistar rats (200-220 g; Vital River Laboratories, Co., Ltd., Beijing, China) were kept in a controlled environment $\left(22-24^{\circ} \mathrm{C}\right.$ and a humidity of $45-55 \%$ with a 12/12 h light/dark cycle, lights on at 7:00 a.m.) on a standard Purina chow diet (4\% fat) with free access to food and water (16). Following acclimatization to the environment for 1 week, rats were randomly divided into 5 groups $(n=6)$ : A normoxia group, a group subjected to $\mathrm{IH}$ with $12.5 \% \mathrm{O}_{2}$, a group subjected to $\mathrm{IH}$ with $10 \% \mathrm{O}_{2}$, a group subjected to $\mathrm{IH}$ with $7.5 \% \mathrm{O}_{2}$ and a group subjected to $\mathrm{IH}$ with $5 \% \mathrm{O}_{2}$. The rats in the latter four groups were subjected to IH with cycles consisting of $60 \mathrm{sec}$ exposure to normal air and $60 \mathrm{sec}$ exposure to hypoxic air between 8:30 a.m. and 4:30 p.m. everyday for 12 weeks. Rats in the normoxia group were exposed to normal air $\left(21 \% \mathrm{O}_{2}\right)$ and acted as a control.

On the first day of the thirteenth week, rats in each group were anesthetized with intraperitoneal injection of $10 \%$ chloral hydrate $(350 \mathrm{mg} / \mathrm{kg}$; (Sinopharm Group, Co., Ltd., Beijing, China) and peripheral blood was collected from the intraperitoneal vein using a disposable syringe through a pinhole incision for all subsequent experiments. Following blood collection, the rats died naturally of blood loss (about $10 \mathrm{ml}$ ), and death was confirmed following respiratory arrest. Pulmonary tissues were harvested from rats, lavaged with sterile saline 3 times, and the bronchoalveolar lavage fluid (BALF) of the 3 times was collected mixed. The experimental protocols and methods of animal sacrifice were line with the experimental standards of Kunming Medical University (Kunming, China) and were approved by the Ethics Committee of Kunming Medical University.

Bradford assay. A Bradford assay was performed to detect the protein concentration of BALF using the Bradford Concentration Detection kit (catalogue no. WLA012; Wanleibio, Shenyang, China). Different concentrations of bovine serum albumin (BSA) standard included in the kit (0, 0.025, 0.05, 0.1, $0.2,0.3,0.4$ and $0.5 \mu \mathrm{g} / \mu \mathrm{l}$ ) were strained with Coomassie Blue $\mathrm{G} 250$ contained in the kit for $5 \mathrm{~min}$ at room temperature and the absorbance was measured at $570 \mathrm{~nm}$ using a microplate reader (BioTek Instruments, Inc., Winnoski, VT, USA) to construct a standard curve. BALF was also strained with G250 and the absorbance was measured at $570 \mathrm{~nm}$. The protein content in the BALF was calculated according to the standard curve.

Giemsa staining. $10 \mu \mathrm{l}$ fresh BALF was dripped onto a glass slide, and a smear was made. The smear was dried at room temperature, fixed with methanol (Sinopharm Group, Co., Ltd., Beijing, China) at room temperature for $15 \mathrm{~min}$ and dried naturally. Subsequently, the smear was stained with Giemsa A (Nanjing Jiancheng Bioengineering Institute, Nanjing, China) for $1 \mathrm{~min}$ and Giemsa B (Nanjing Jiancheng Bioengineering Institute) for $7 \mathrm{~min}$, destained with $80 \%$ ethanol (Sinopharm Group, Co., Ltd.) and washed with water. The smear was dried at room temperature and then observed using a light microscope (magnification, x600; Olympus Corp., Tokyo, Japan). The numbers of total leukocytes, monocytes and polykaryocytes in the BALF were counted in 6 fields in each group and average values were calculated.

Hematoxylin and eosin $(H \& E)$ staining. H\&E staining was performed to detect pulmonary tissue lesions. Pulmonary tissues from the rats were fixed with $4 \%$ paraformaldehyde (Sinopharm Group, Co., Ltd.) at room temperature overnight, washed for $6 \mathrm{~h}$ with water and dehydrated in graded concentrations of ethanol (70\% for $2 \mathrm{~h}, 80 \%$ overnight, $90 \%$ for $2 \mathrm{~h}$ and $100 \%$ for $1 \mathrm{~h}$, twice). Tissues were immersed in xylene (Sinopharm Group, Co., Ltd.) until they were transparent and then embedded in paraffin at $60^{\circ} \mathrm{C}$ for $2 \mathrm{~h}$. The tissue block was cut into $5 \mu \mathrm{m}$ pieces, spread in water and dried on glass slide at $60^{\circ} \mathrm{C}$ for $24 \mathrm{~h}$. Slices were dewaxed in xylene, hydrated in graded concentrations of ethyl alcohol (100\% for $5 \mathrm{~min}$, twice, $95 \%$ for $2 \mathrm{~min}, 85 \%$ for $2 \mathrm{~min}, 75 \%$ for $2 \mathrm{~min}$ ) and water for $2 \mathrm{~min}$. The slices were then stained with hematoxylin (Beijing Solarbio Science \& Technology, Co., Ltd., Beijing, China) for 5 min and eosin (Sinopharm Group, Co., Ltd.) for 3 min, dehydrated in graded concentrations of ethanol $(75,85,95 \%$ for 2 min each time and $100 \%$ twice for 5 min each time), dehydrated in xylene twice for $10 \mathrm{~min}$ and mounted with neutral balsam. Sections were observed and photographed using a light microscope (magnification, x200; Olympus Corp.).

Detection of malondialdehyde (MDA), superoxide dismutase (SOD) and glutathione (GSH) levels. The tissue was added to a 9-fold volume of PBS, dispersed to the homogenate with a homogenizer, freeze-thawed 3 times, and centrifuged at $14,937 \mathrm{x} \mathrm{g}$ at $4^{\circ} \mathrm{C}$ for $10 \mathrm{~min}$. The supernatant was collected as a protein sample and protein concentration was detected using a BCA Protein Quantitative Kit (Wanleibio) according to the manufacturer's protocol. MDA content in the serum was detected using the MDA Assay kit (catalogue no. A003-1), SOD activity was detected by the SOD Assay kit (catalogue no. A001-3) and GSH content was detected using the GSH Assay kit (catalogue no. A006-2; all Nanjing Jiancheng Bioengineering Institute) following the manufacturer's protocols.

Serum enzyme-linked immunosorbent assay (ELISA). The collected blood was solidified at room temperature for $20 \mathrm{~min}$ and centrifuged at $1,028 \mathrm{x}$ g at $4^{\circ} \mathrm{C}$ for $20 \mathrm{~min}$. The supernatant was collected to detect $\mathrm{C}$-reactive protein (CRP) and 8-isoprostane. CRP content in the serum was detected using the rat CRP ELISA kit (catalogue no. EK0978; Wuhan Boster Biological Technology, Ltd., Wuhan, China) according to the manufacturers' protocol. The 8 -isoprostane content in the serum was detected using the rat 8-iso-PG ELISA kit (catalogue no. DRE00754; Shanghai WHB Biotech, Co., Ltd., Shanghai, China) according to the manufacturer's instructions.

Tissue ELISA. Tissue was added to PBS, scattered to cell suspension with a homogenizer, freeze-thawed 3 times and centrifuged at $14,937 \mathrm{x} g$ at $4^{\circ} \mathrm{C}$ for $10 \mathrm{~min}$. The supernatant was collected as the protein sample and protein concentration was detected using the BCA Protein Quantitative Kit according to the manufacturer's protocol. 
Tumor necrosis factor- $\alpha(\mathrm{TNF}-\alpha)$ levels were detected using a rat TNF- $\alpha$ ELISA kit (catalogue no. EK0526) and interleukin (IL)-1 $\beta$ content was detected using the rat IL-1 $\beta$ ELISA kit (catalogue no. EK0393) and IL-6 content was detected using the rat IL-6 ELISA kit (catalogue no. EK0412; all Wuhan Boster Biological Technology, Ltd.), following the manufacturer's protocols.

Western blotting. Total protein was extracted from tissues using a Whole Cell Lysis Assay Kit (Wanleibio) and proteins from the cell nuclei were extracted using the Nucleus Protein Extraction kit (Wanleibio) according to the manufacturer's protocols. Following determination of protein concentration using the BCA Protein Quantitative kit, total proteins and nuclei proteins were separated by SDS-PAGE; stacking gel of $5 \%$, the separating gel of inducible nitric oxidate synthase (iNOS), cyclooxygenase (COX)-2 and nuclear factor erythroid 2-related factor 2 (Nrf2) was $10 \%$ and the separating gel of heme oxygenase (HO)-1 was $13 \%$. Proteins and nuclei proteins were transferred to a PVDF membrane (EMD Millipore, Billerica, MA, USA). Following blocking with 5\% skim milk (Yili Group, Hohhot, China) at room temperature for $1 \mathrm{~h}$, the PVDF membrane was incubated with the following antibodies at $4{ }^{\circ} \mathrm{C}$ overnight: Rabbit anti- COX-2 (1:400; catalogue no. BA0738), rabbit anti- iNOS; (1:400; catalogue no. BA0362; both Wuhan Boster Biological Technology, Ltd.), rabbit anti- HO-1 (1:200; catalogue no. sc-10789; Santa Cruz Biotechnology, Inc., Dallas, TX, USA) and rabbit anti- Nrf2 (1:400; catalogue no. BA3790; Wuhan Boster Biological Technology, Ltd.). Subsequently, the PVDF membrane was washed with TBST 4 times for $5 \mathrm{~min}$, and incubated with horseradish peroxidase (HRP)-conjugated goat anti-rabbit immunoglobulin (Ig) G (1:5,000; catalogue no. WLA023; Wanleibio) and exposed to an ECL Reagent Kit (Wanleibio). $\beta$-actin was used as the internal control of COX-2, iNOS and HO-1, and histone $\mathrm{H} 3$ as the control of mitochondrial control of Nrf2. Thereafter, antibodies were removed using a Western Blot Striping Buffer (Wanleibio) and the PVDF membrane was incubated with mouse anti- $\beta$-actin $(1: 1,000$; catalogue no. sc-47778, Santa Cruz Biotechnology, Inc.) and goat anti-mouse IgG-HRP (1:5,000; catalogue no. WLA024, Wanleibio) to detect the internal control of total protein, $\beta$-actin, or incubated with rabbit anti-histone H3 $(1: 1,000$; catalogue no. bs-17422R Santa Cruz Biotechnology, Inc.) and goat anti-rabbit IgG-HRP (1:5,000; catalogue no. WLA023, Wanleibio) to detect the internal control of nucleus protein, histone H3. The bands were analyzed with Gel-Pro-Analyzer software, version 4 (Media Cybernetics, Rockville, MD, USA).

Immunohistochemistry. Pulmonary tissues were fixed with $4 \%$ paraformaldehyde at room temperature overnight, rinsed with water for $4 \mathrm{~h}$, dehydrated with graded concentrations of ethanol (70, 80, 90 and 100\%) and hyalinized with xylene. Tissues were paraffin-embedded, sliced into $5 \mu \mathrm{m}$ sections and dried on glass slides at $60^{\circ} \mathrm{C}$. Sections were dewaxed with xylene for 15 min twice and rehydrated with ethanol (95, 85 and $75 \%, 2 \mathrm{~min}$ each) and water for $2 \mathrm{~min}$. Subsequently, sections were incubated with antigen retrieval buffer $(1.8 \mathrm{mM}$ citric acid, $8.2 \mathrm{mM}$ sodium citrate) at $100^{\circ} \mathrm{C}$ for $10 \mathrm{~min}$. Following blocking with $3 \% \mathrm{H}_{2} \mathrm{O}_{2}$ (Sinopharm Group, Co., Ltd.) at room temperature for $15 \mathrm{~min}$ and goat serum (Beijing
Solarbio Science \& Technology, Co., Ltd.) at room temperature for $15 \mathrm{~min}$, sections were incubated with primary rabbit anti-COX-2 (1:200) or rabbit anti-iNOS (1:200) antibodies at $4^{\circ} \mathrm{C}$ overnight. Following rinsing with PBS, sections were incubated with goat anti-rabbit IgG labeled with biotin (1:200; Beyotime Institute of Biotechnology, Haimen, China) for $30 \mathrm{~min}$ at $37^{\circ} \mathrm{C}$. Subsequently, sections were incubated with HRP-labeled streptavidin (Beyotime Institute of Biotechnology) for $30 \mathrm{~min}$ at $37^{\circ} \mathrm{C}$ and DBA color development buffer (Beyotime Institute of Biotechnology) and strained with hematoxylin reagent for $3 \mathrm{~min}$. Subsequently, sections were soaked in $1 \%$ hydrochloric/alcohol for $3 \mathrm{sec}$, washed with water for 20 min, dehydrated with 75, 85, 95\% ethanol for 2 min each time and $100 \%$ ethanol for 5 min twice, and hyalinized with xylene for 5 min twice. Finally, following removal of any residual liquid, half a drop of neutral gum was added onto the section, which was then covered with a cover slip and dried at room temperature. Sections were photographed using a light microscope (magnification, x400; Olympus Corporation).

Statistical analysis. The data in the present study was analyzed with the GraphPad Prism version 5.01 software (GraphPad Software, Inc., La Jolla, CA, USA). The results of the current study are presented as the mean \pm standard deviation of three individual experiments. Data were analyzed using one-way analysis of variance, followed by Dunn's Multiple Comparison Test, and $\mathrm{P}<0.05$ was considered to indicate a statistically significant difference.

\section{Results}

Establishment of the rat OSA model via IH. To determine the effect of OSA on pulmonary tissue, a rat OSA model was established by performing IH for 12 weeks. As the clinical severity of patients with OSA differs, different oxygen concentrations were used to induce $\mathrm{IH}: 12.5 \% \mathrm{O}_{2}, 10 \% \mathrm{O}_{2}, 7.5 \% \mathrm{O}_{2}$ and $5 \% \mathrm{O}_{2}$. Control mice were exposed to room air containing $21 \% \mathrm{O}_{2}$ throughout the experiment. Total protein content of BALF was measured using a Bradford assay and leukocyte, monocyte and polykaryocyte numbers were determined using Giemsa staining.

IH led to a significant increase in the total protein content of BALF $(\mathrm{P}<0.05)$, with greater increases in protein content detected at lower oxygen concentrations (Fig. 1A). Giemsa staining that IH increased leukocyte, monocyte and polykaryocyte numbers in BALF in a concentration-dependent manner. Treatment with $5 \% \quad \mathrm{O}_{2}$ increased leukocyte numbers by 2.25-fold ( $\mathrm{P}<0.001$; Fig. 1B), monocyte numbers by 6.21 -fold $(\mathrm{P}<0.001$; Fig. 1C) and polykaryocyte numbers by 3.29 -fold $(\mathrm{P}<0.001$; Fig. 1D), respectively, compared with the normoxic group. H\&E staining indicated that, following IH treatment, the pulmonary alveoli were broken, a large number of inflammatory cells appeared, the number of necrotic and apoptotic cells increased and edema was identified in the pulmonary tissues (Fig. 1E), suggesting pulmonary inflammation. There was a negative association between the severity of pulmonary injury and oxygen concentration.

OSA causes an oxidative stress response and pulmonary inflammation. As IH may cause an oxidative stress response, 
A

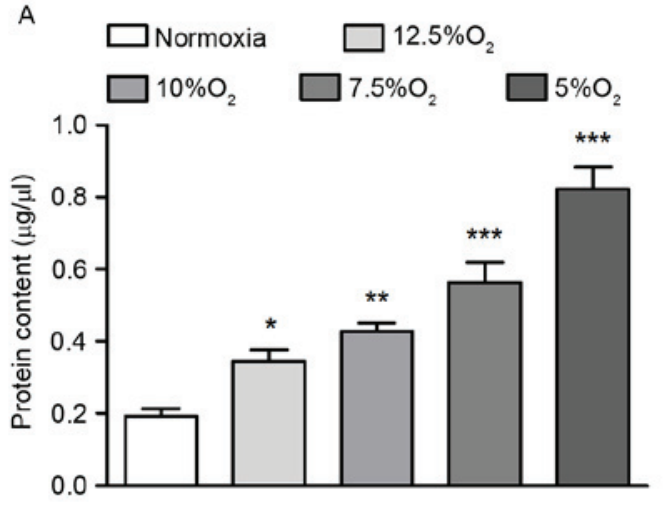

C

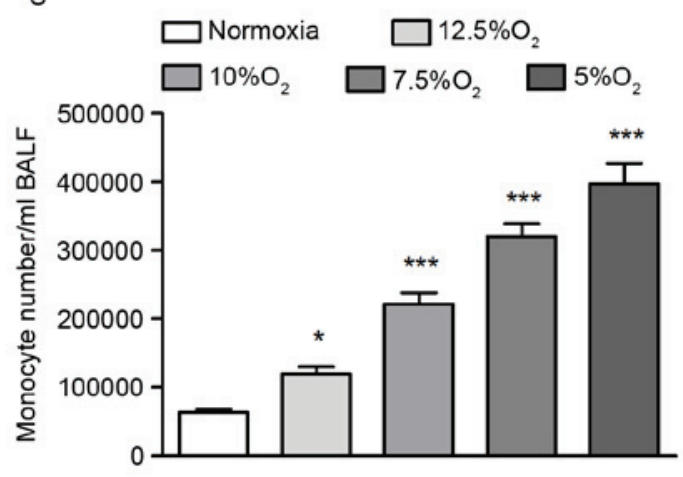

B

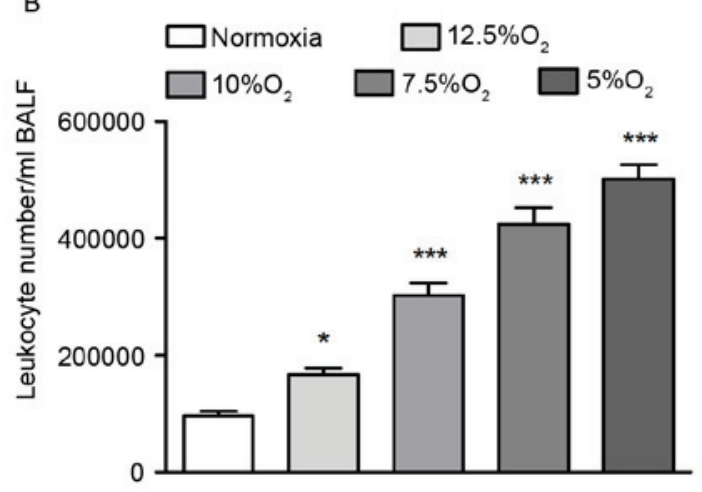

D

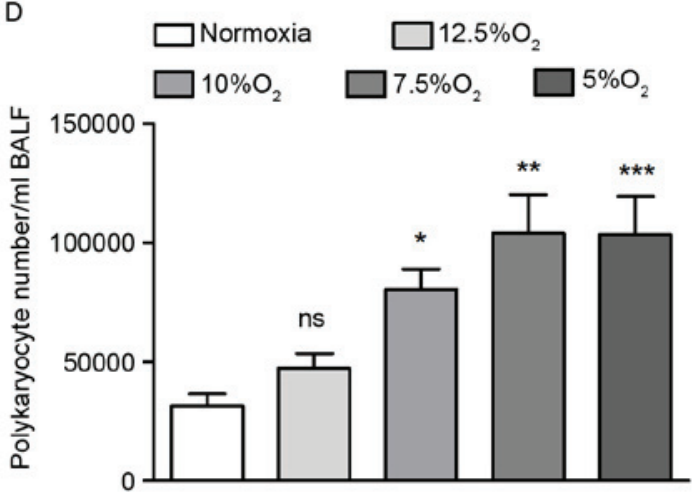

$\mathrm{E}$

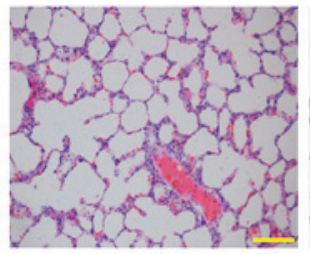

Normoxia

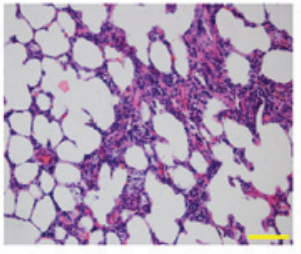

$12.5 \% \mathrm{O}_{2}$

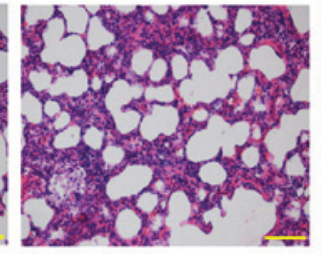

$10 \% \mathrm{O}_{2}$

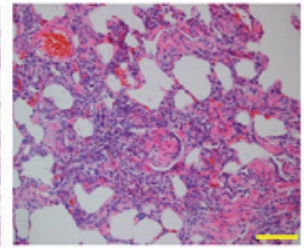

$7.5 \% \mathrm{O}_{2}$

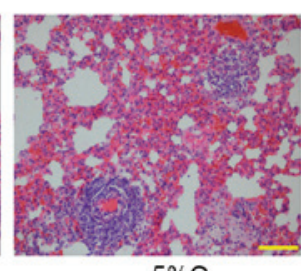

$5 \% \mathrm{O}_{2}$

Figure 1. Establishment of the rat obstructive sleep apnea model. (A) Protein content of BALF was detected by Bradford assay. (B) Leukocyte numbers in the BALF of rats at different concentrations of $\mathrm{O}_{2}$. (C) Monocyte numbers in the BALF of rats at different concentrations of $\mathrm{O}_{2}$. (D) Polykaryocyte numbers in the BALF of rats at different concentrations of $\mathrm{O}_{2}$. (E) Hematoxylin and eosin staining of rat pulmonary tissues from each group. Scale bar=100 $\mu \mathrm{m}$. ${ }^{*} \mathrm{P}<0.05$, ${ }^{* *} \mathrm{P}<0.01,{ }^{* * *} \mathrm{P}<0.001$ vs. normoxia. Ns, no significance; BALF, bronchoalveolar lavage fluid.

several markers of the oxidative stress response, including CRP and 8-isoprostane in the serum, as well as MDA, SOD and GSH in the pulmonary tissue, were measured. Following a decrease in oxygen concentration from 12.5 to $5 \%$, CRP levels in the serum increased by $2.71,4.19,6.32$ and 8.52 -fold (Fig. 2A) and 8-isoprotane levels in the serum increased by 1.81,2.72, 3.23 and 4.38 -fold (Fig. 2B), respectively. These increases in CRP and 8-isoprotane levels were significant at $\mathrm{O}_{2}$ levels $\leq 10 \%$ $(\mathrm{P}<0.01$; Fig. $2 \mathrm{~A}$ and B). Furthermore, following IH treatment with 12.5, 10, 7.5 and $5 \% \mathrm{O}_{2}$, MDA content in the pulmonary tissue increased by 1.34, 1.62, 2.15 and 3.98-fold (Fig. 2C), SOD activity decreased by 29, 37, 50 and $63 \%$ (Fig. 2D) and GSH content decreased by 10, 19, 42 and 51\%, (Fig. 2E) respectively, compared with the normoxic group. These differences were most significant following treatment with $5 \% \mathrm{O}_{2}(\mathrm{P}<0.001$; Fig. 2C-E), indicating that a marked oxidative stress response occurred. In addition, levels of several inflammation factors, including TNF- $\alpha$, IL- $1 \beta$ and IL- 6 in the pulmonary tissue were detected by ELISA. The results demonstrated that IH increased TNF- $\alpha$, IL-1 $\beta$ and IL- 6 levels in a concentration-dependent manner, with the most significant increases occurring following treatment with $5 \% \mathrm{O}_{2}(\mathrm{P}<0.001 ;$ Fig. 2F-H $)$.

OSA increases expression of COX-2 and iNOS. Subsequently, the expression of two enzymes, COX-2 and iNOS, that serve crucial roles in the oxidative stress response and inflammation, were detected by western blotting and immunohistochemistry. The results of western blotting demonstrated that $\mathrm{IH}$ with 12.5 , $10,7.5$ and $5 \% \mathrm{O}_{2}$ increased the expression of COX-2 by 1.34 , 2.3, 2.79 and 3.05-fold (Fig. 3A) and increased the expression of iNOS by 1.18, 1.99, 3.78 and 5.93-fold in pulmonary tissue (Fig. 3B). Significant increases in COX-2 expression occurred following treatment with $\leq 10 \% \mathrm{O}_{2}(\mathrm{P}<0.001$; Fig. 3A) and significant increases in iNOS expression occurred following treatment with $\leq 7.5 \% \mathrm{O}_{2}(\mathrm{P}<0.001$; Fig. 3B $)$. The results from immunohistochemistry confirmed that the expression of 

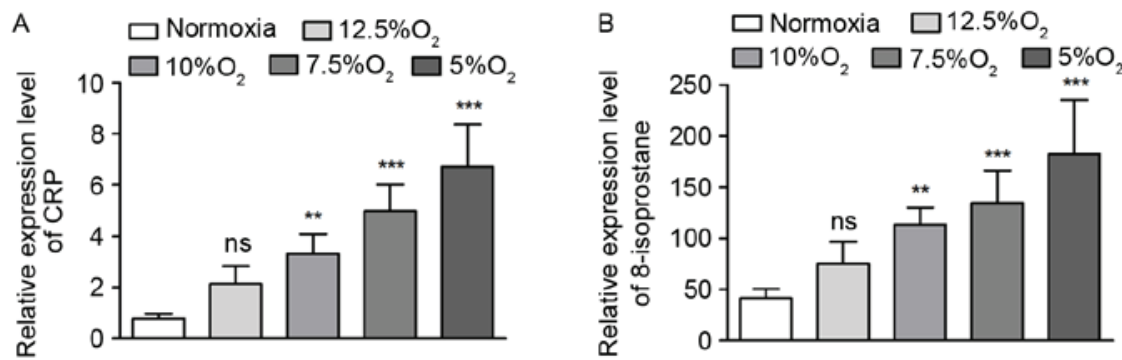

C
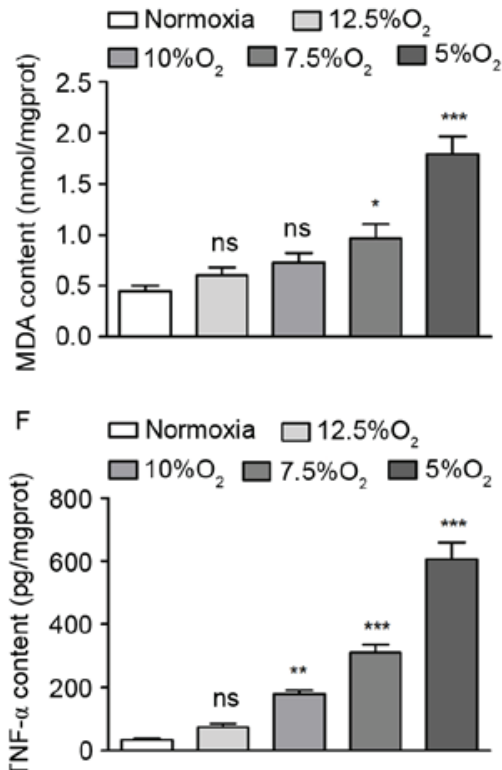

D

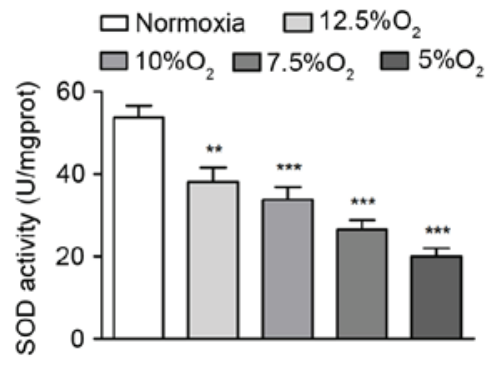

G

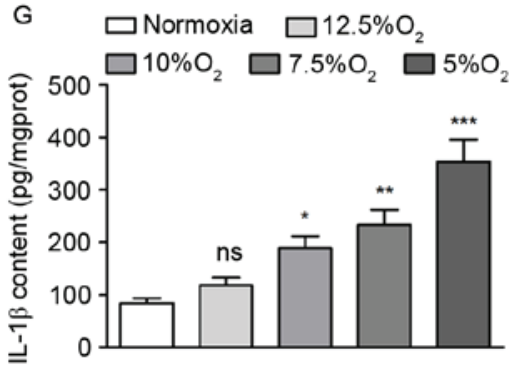

$E$

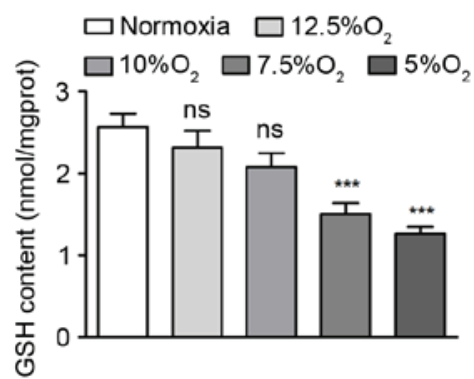

$\mathrm{H}$
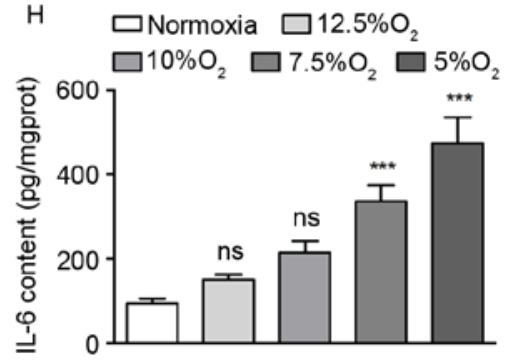

Figure 2. Obstructive sleep apnea causes an oxidative stress response and pulmonary inflammation. (A) CRP levels in the serum. (B) Levels of 8-isoprostane in the serum. (C) MDA content in rat pulmonary tissues. (D) SOD activity in the pulmonary tissues of rats. (E) GSH content in the pulmonary tissues of rats. (F) The expression of TNF- $\alpha$ in the pulmonary tissues of rats. (G) The expression IL-1 $\beta$ in the pulmonary tissues of rats. (H) The expression of IL-6 in the pulmonary tissues of rats. ${ }^{*} \mathrm{P}<0.05,{ }^{* *} \mathrm{P}<0.01,{ }^{* * *} \mathrm{P}<0.001$ vs. normoxia. CRP, C reactive protein; MDA, malondialdehyde; SOD, superoxide dismutase; GSH, glutathione; TNF, tumor necrosis factor; IL, interleukin; ns, no significance.

COX-2 and iNOS increased with decreasing concentrations of oxygen (Figs. 3C and D).

OSA activates the Nrf2/HO-1 signaling pathway. Nrf2/HO-1 is an antioxidant signaling pathway, therefore the expression of Nrf2 and HO-1 in pulmonary tissue following IH treatment was measured. The results from western blotting demonstrated that IH increased the expression of HO-1 by 1.03, 2.95, 2.82 and 2.64-fold (Fig. 4A) These increases were significant following treatment with $\leq 10 \% \mathrm{O}_{2}(\mathrm{P}<0.001$; Fig. 4A). Under normal conditions, Nrf2 is distributed in the cytoplasm. However, during oxidative stress, Nrf2 enters the nucleus to regulate its downstream genes at the transcriptional level and activates the oxidative stress response (17). Therefore, levels of Nrf2 in the nucleus itself were detected by western blotting and it was demonstrated that Nrf2 levels in the nucleus increased by 1.83 , 2.45 and 1.88-fold following IH treatment with 10, 7.5 and 5\% $\mathrm{O}_{2}$, a significant increase $(\mathrm{P}<0.01$; Fig. 4B).

Taken together, these results demonstrate that in rats, OSA simulated by IH caused an oxidative stress response and pulmonary inflammation, and activated the canonical antioxidative Nrf2/HO-1 signaling pathway in a dose-dependent manner. These results may be important in identifying novel treatments for pulmonary damage caused by OSA.

\section{Discussion}

In the present study, a rat model of OSA of different degrees was established by $\mathrm{IH}$ treatment with $12.5,10,7.5$ and $5 \% \mathrm{O}_{2}$ over 12 weeks for $8 \mathrm{~h}$ per day. Following treatment, a number of oxidative stress and inflammatory factors were detected.

CRP is an acute phase response protein and its levels rapidly increase following inflammation. Its expression is stimulated by IL-6 at the transcriptional level (18). 8-isoprotane is a product of the peroxidation of arachidonic acid (AA) on the cell membrane, which is catalyzed by reactive oxygen species (ROS). It damages the cell membrane and is considered to be a marker of oxidative stress (19). MDA is the final production of lipid oxidation catalyzed by ROS, which attacks the cell membrane and the electron transport chain (20). Therefore in the current study, levels of CRP, 8-isoprotane and MDA were measured as biomarkers of the oxidative stress response. SOD is an important anti-oxidant enzyme that, together with catalase and peroxidase, eliminates ROS (21). GSH is a tripeptide, which is oxidized by ROS to glutathione disulfide (GSSG) in a reaction catalyzed by glutathione peroxidase (GPx). GSSG is then reduced by gluthathione reductase (GR) to GSH to eliminate ROS (22). SOD and GSH, which serve crucial roles in eliminating high levels of ROS, may be consumed 
A

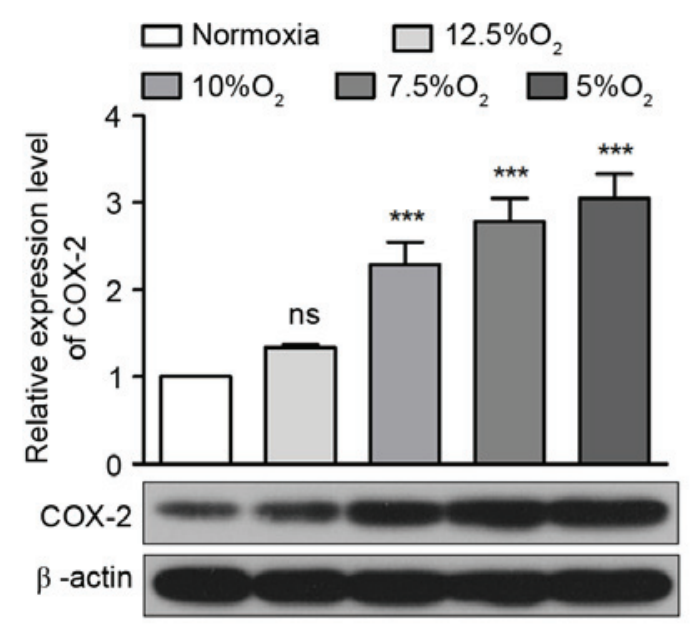

B

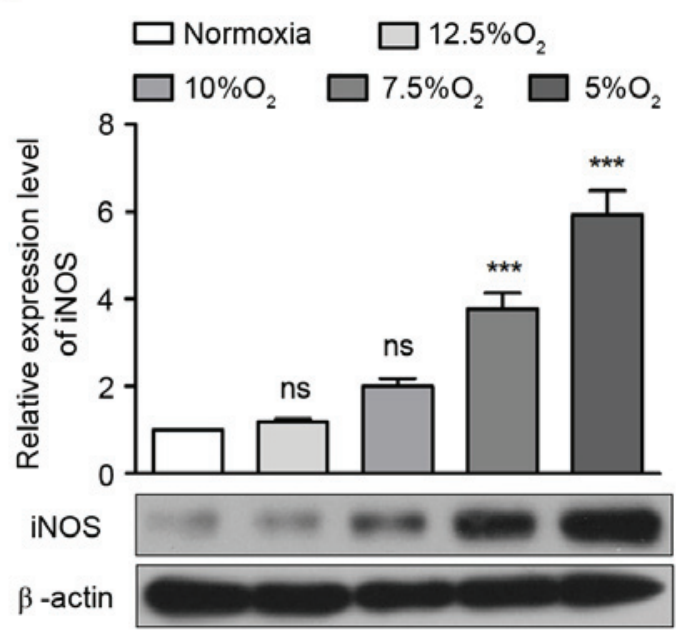

C

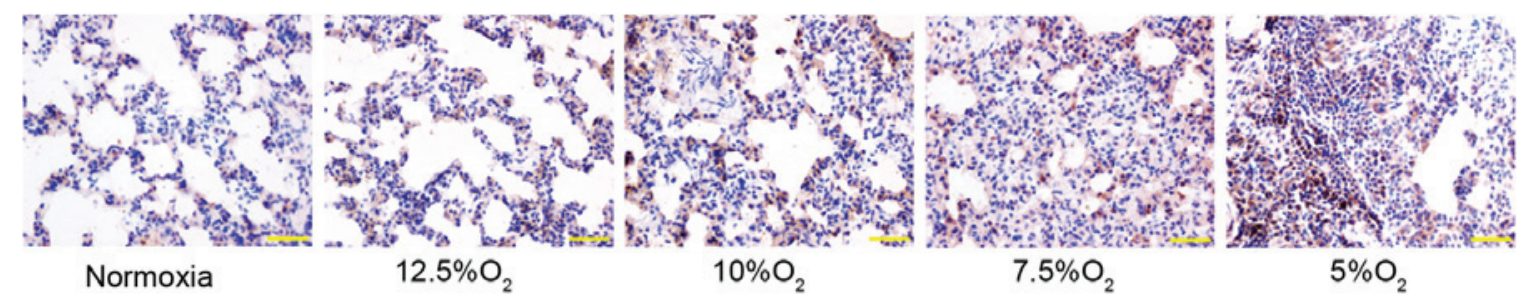

D

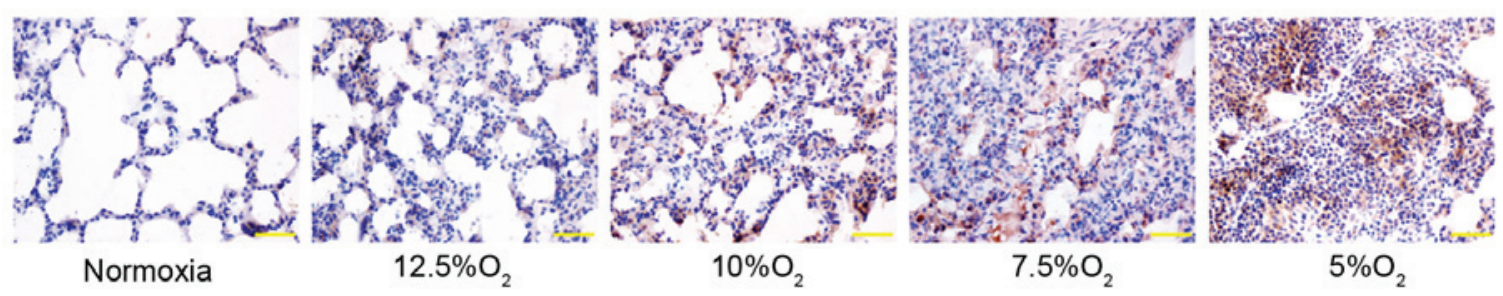

Figure 3. Obstructive sleep apnea increases expression of COX-2 and iNOS. (A) The expression of COX-2 in pulmonary tissue of rats detected by western blotting. (B) The expression of iNOS in the pulmonary tissue of rats detected by western blotting. (C) Immunohistochemical detection of anti-COX-2 in the pulmonary tissue of rats. (D) Immunohistochemical detection of anti-iNOS in the pulmonary tissue of rats. Scale bar=50 $\mu \mathrm{m}$. ${ }^{* * *} \mathrm{P}<0.001 \mathrm{vs}$. normoxia. iNOS, inducible nitric oxide synthase; COX-2, cyclooxygenase; ns, no significance.
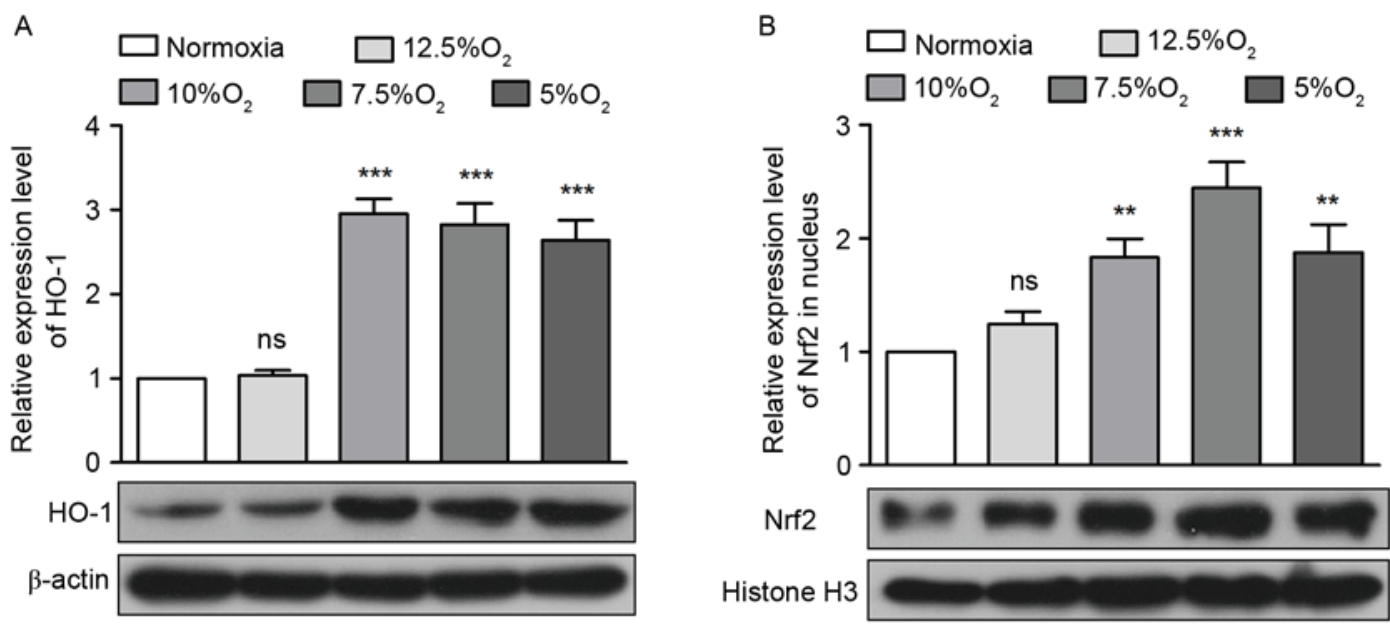

Figure 4. Obstructive sleep apnea activates the Nrf2/HO-1 signaling pathway. (A) The expression of HO-1 in the pulmonary tissue of rats as detected by western blotting. (B) The expression of Nrf2 in the pulmonary tissue nuclei of rats as detected by western blotting. ${ }^{* * *} \mathrm{P}<0.01,{ }^{* * *} \mathrm{P}<0.001$ vs. normoxia. HO-1, heme oxygenase 1; Nrf2, nuclear factor erythroid 2-related factor 2; ns, no significance. 
by a large quantity of ROS (23). In the current study, it was demonstrated that IH causes an oxidative stress response, producing large amounts of ROS, thus causing an increase in CRP, 8-isoprotane and MDA and decreases in SOD and GSH. The expression of TNF- $\alpha$, IL-1 $\beta$ and IL-6 in pulmonary tissue subsequently increased. TNF- $\alpha$, IL- $1 \beta$ and IL- 6 are all produced by leukocytes and serve important roles in inflammatory responses; these increases indicated the presence of inflammation $(24,25)$.

In addition, $\mathrm{IH}$ activates hypoxia induced factor $\alpha$ that stimulates the transcription of various genes, including iNOS and COX-2 (26,27). During oxidative stress, the expression of iNOS increases rapidly and produces higher levels of nitric oxide (NO) than during normoxia. NO is an important messenger molecule in the inflammatory response (28). iNOS expression is induced by a number of cellular factors, including interferon $\gamma$, IL-1, IL-6 and TNF- $\alpha$ (29). COX is a key enzyme in the synthesis of prostaglandin and COX-2 is an inducible isomer (30). In the presence of high levels of ROS, COX-2 catalyzes the oxidation of AA into prostaglandin, which consequently participates in inflammatory processes. Furthermore, COX-2 catalyzes the oxidation of docosahexaenoic acid, eicosapentaenoic acid and docodapentaenoid acid into electrophilic oxoderivatives (EFOXs), which exhibit anti-inflammatory and antioxidant properties (31). Most notably, EFOXs are able to activate a crucial molecule, Nrf2, in the oxidative stress response (31).

Nrf2, a member of the basic leucine zipper transcription factor family, is the most important regulator of antioxidant defenses (32). Under normoxic conditions, Nrf2 is sequestered in the cytoplasm by binding to Kelch-like ECH-associated protein 1 (Keap 1), which anchors to the actin cytoskeleton, promoting ubiquitination and eventual degradation of Nrf2 $(33,34)$. Keap 1 is a cysteine-rich protein and may be modified by electrophiles. Under conditions of oxidative stress, EFOXs oxidize crucial cysteines of Keap1, leading to the release and stabilization of Nrf2. Accumulated Nrf2 then translocates into the nucleus, activates the antioxidant response element via Maf $(35,36)$ and promotes the transcription of downstream genes, including GR, GPx, gluthathione S-tranferase, $\gamma$-glutamylcysteine ligase, SOD, NAD(P) $\mathrm{H}$ :quinine dehydrogenase (NQO1) and HO-1 (37), which are all crucial in the antioxidative and anti-inflammatory responses.

HO-1, also known as heat shock protein 32 (HSP32), is an inducible enzyme that is induced by multiple stresses. HO-1 catalyzes heme to generate biliverdin, ferrous ion $\left(\mathrm{Fe}^{2+}\right)$ and carbon monoxide (CO) (38). $\mathrm{CO}$ is a vital signal mediator in multiple signaling pathways of anti-inflammatory and cardioprotective capacities under conditions of oxidative stress $(39,40)$. Biliverdin may be reduced to bilirubin, which is a potent antioxidant and protects cells from oxidative stress (41).

In conclusion, the current study demonstrated that $\mathrm{IH}$ increases the expression of COX-2 and iNOS and subsequently activates $\mathrm{Nrf} 2$. The nuclear translocation of $\mathrm{Nrf} 2$ accelerates the transcription of downstream genes, such as HO-1, to attenuate the inflammation caused by IH in the pulmonary tissue of rats reliant on a reliable oxygen supply. These results may be of great significance in the diagnosis and therapy of OSA.

\section{Acknowledgements}

The present study was supported by a grant from the National Natural Science Foundation of China (no. 81460017).

\section{References}

1. Khoury J and Doghramji K: Primary sleep disorders. Psychiatr Clin North Am 38: 683-704, 2015.

2. Franklin KA and Lindberg E: Obstructive sleep apnea is a common disorder in the population-a review on the epidemiology of sleep apnea. J Thorac Dis 7: 1311-1322, 2015.

3. Rozova K and Mankovska I: The effect of intermittent hypoxic training on lung and heart tissues of healthy rats. Pneumonol Alergol Pol 80: 296-300, 2012.

4. da Rosa DP, Forgiarini LF, Baronio D, Feijó CA, Martinez D and Marroni NP: Simulating sleep apnea by exposure to intermittent hypoxia induces inflammation in the lung and liver. Mediators Inflamm 2012: 879419, 2012.

5. Yaggi HK, Concato J, Kernan WN, Lichtman JH, Brass LM and Mohsenin V: Obstructive sleep apnea as a risk factor for stroke and death. N Engl J Med 353: 2034-2041, 2005.

6. Munoz R, Duran-Cantolla J, Martínez-Vila E, Gallego J, Rubio R, Aizpuru F and De La Torre G: Severe sleep apnea and risk of ischemic stroke in the elderly. Stroke 37: 2317-2321, 2006.

7. Lavie P, Ben-Yosef R and Rubin AE: Prevalence of sleep apnea syndrome among patients with essential hypertension. Am Heart J 108: 373-376, 1984.

8. Mehra R, Benjamin EJ, Shahar E, Gottlieb DJ, Nawabit R, Kirchner HL, Sahadevan J and Redline S; Sleep Heart Health S: Association of nocturnal arrhythmias with sleep-disordered breathing: The sleep heart health study. Am J Respir Crit Care Med 173: 910-916, 2006.

9. Gottlieb DJ, Yenokyan G, Newman AB, O'Connor GT, Punjabi NM, Quan SF, Redline S, Resnick HE, Tong EK, Diener-West $M$ and Shahar E: Prospective study of obstructive sleep apnea and incident coronary heart disease and heart failure: The sleep heart health study. Circulation 122: 352-360, 2010.

10. Coughlin SR, Mawdsley L, Mugarza JA, Calverley PM and Wilding JP: Obstructive sleep apnoea is independently associated with an increased prevalence of metabolic syndrome. Eur Heart J 25: 735-741, 2004.

11. Jun JC, Chopra S and Schwartz AR: Sleep apnoea. Eur Respir Rev 25: 12-18, 2016.

12. Mermigkis C, Bouloukaki I, Antoniou K, Papadogiannis G, Giannarakis I, Varouchakis G, Siafakas N and Schiza SE: Obstructive sleep apnea should be treated in patients with idiopathic pulmonary fibrosis. Sleep Breath 19: 385-391, 2015.

13. Tamisier R, Tan CO, Pepin JL, Levy P and Taylor JA: Blood pressure increases in OSA due to maintained neurovascular sympathetic transduction: Impact of CPAP. Sleep 38: 1973-1980, 2015.

14. Gonchar O and Mankovska I: Moderate hypoxia/hyperoxia attenuates acute hypoxia-induced oxidative damage and improves antioxidant defense in lung mitochondria. Acta Physiol Hung 99: 436-446, 2012.

15. Almendros I, Montserrat JM, Torres M, Dalmases M, Cabañas ML, Campos-Rodríguez F, Navajas D and Farré R: Intermittent hypoxia increases melanoma metastasis to the lung in a mouse model of sleep apnea. Respir Physiol Neurobiol 186: 303-307, 2013

16. Savransky V, Nanayakkara A, Vivero A, Li J, Bevans S, Smith PL, Torbenson MS and Polotsky VY: Chronic intermittent hypoxia predisposes to liver injury. Hepatology 45: 1007-1013, 2007.

17. Bruns DR, Drake JC, Biela LM, Peelor FF III, Miller BF and Hamilton KL: Nrf2 signaling and the slowed aging phenotype: Evidence from long-lived models. Oxid Med Cell Longev 2015: 732596, 2015.

18. Pepys MB and Hirschfield GM: C-reactive protein: A critical update. J Clin Invest 111: 1805-1812, 2003.

19. Praticò D, Lawson JA, Rokach J and FitzGerald GA: The isoprostanes in biology and medicine. Trends Endocrinol Metab 12: 243-247, 2001.

20. Tian XH, Liu CL, Jiang HL, Zhang Y, Han JC, Liu J and Chen M: Cardioprotection provided by Echinatin against ischemia/reperfusion in isolated rat hearts. BMC Cardiovasc Disord 16: 119, 2016. 
21. Zhao Y, Zhao E, Zhang $\mathrm{C}$ and Zhang H: Study of the changes of acrosomal enzyme, nitric oxide synthase, and superoxide dismutase of infertile patients with positive antisperm antibody in seminal plasma. Cell Biochem Biophys 73: 639-642, 2015.

22. Jacob C, Giles GI, Giles NM and Sies H: Sulfur and selenium: The role of oxidation state in protein structure and function. Angew Chem Int Ed Engl 42: 4742-4758, 2003.

23. Valko M, Jomova K, Rhodes CJ, Kuča K and Musílek K: Redoxand non-redox-metal-induced formation of free radicals and their role in human disease. Arch Toxicol 90: 1-37, 2016.

24. Ma K, Zhang $\mathrm{H}$ and Baloch Z: Pathogenetic and therapeutic applications of tumor necrosis factor- $\alpha$ (TNF- $\alpha$ ) in major depressive disorder: A systematic review. Int J Mol Sci 17: pii: E733, 2016.

25. Dmitrieva OS, Shilovskiy IP, Khaitov MR and Grivennikov SI: Interleukins 1 and 6 as main mediators of inflammation and cancer. Biochemistry (Mosc) 81: 80-90, 2016.

26. Robinson MA, Baumgardner JE and Otto CM: Oxygen-dependent regulation of nitric oxide production by inducible nitric oxide synthase. Free Radic Biol Med 51: 1952-1965, 2011.

27. Csiki I, Yanagisawa K, Haruki N, Nadaf S, Morrow JD, Johnson DH and Carbone DP: Thioredoxin-1 modulates transcription of cyclooxygenase-2 via hypoxia-inducible factor-1alpha in non-small cell lung cancer. Cancer Res 66: 143-150, 2006.

28. Guzik TJ, Korbut R and Adamek-Guzik T: Nitric oxide and superoxide in inflammation and immune regulation. J Physiol Pharmacol 54: 469-487, 2003.

29. Kleinert H, Pautz A, Linker K and Schwarz PM: Regulation of the expression of inducible nitric oxide synthase. Eur J Pharmacol 500: 255-266, 2004.

30. Luo C, Strauss L, Ristimäki A, Streng T and Santti R: Constant expression of cyclooxygenase- 2 gene in prostate and the lower urinary tract of estrogen-treated male rats. Z Naturforsch C 56 : 455-463, 2001

31. Chen C: COX-2's new role in inflammation. Nat Chem Biol 6 : 401-402, 2010
32. Alfieri A, Srivastava S, Siow RC, Modo M, Fraser PA and Mann GE: Targeting the Nrf2-Keap1 antioxidant defence pathway for neurovascular protection in stroke. J Physiol 589: 4125-4136, 2011

33. Kang MI, Kobayashi A, Wakabayashi N, Kim SG and Yamamoto M: Scaffolding of Keap1 to the actin cytoskeleton controls the function of Nrf2 as key regulator of cytoprotective phase 2 genes. Proc Natl Acad Sci USA 101: 2046-2051, 2004.

34. Kobayashi M and Yamamoto M: Nrf2-Keap1 regulation of cellular defense mechanisms against electrophiles and reactive oxygen species. Adv Enzyme Regul 46: 113-140, 2006.

35. Kobayashi A, Kang MI, Watai Y, Tong KI, Shibata T, Uchida K and Yamamoto M: Oxidative and electrophilic stresses activate Nrf2 through inhibition of ubiquitination activity of Keap1. Mol Cell Biol 26: 221-229, 2006.

36. Canning P, Sorrell FJ and Bullock AN: Structural basis of Keap1 interactions with Nrf2. Free Radic Biol Med 88: 101-107, 2015.

37. Loboda A, Damulewicz M, Pyza E, Jozkowicz A and Dulak J: Role of Nrf2/HO-1 system in development, oxidative stress response and diseases: An evolutionarily conserved mechanism. Cell Mol Life Sci 73: 3221-3247, 2016.

38. Kozakowska M, Dulak J and Józkowicz A: Heme oxygenase-1 more than the cytoprotection. Postepy Biochem 61: 147-158, 2015 (In Polish).

39. Morse D, Pischke SE, Zhou Z, Davis RJ, Flavell RA, Loop T, Otterbein SL, Otterbein LE and Choi AM: Suppression of inflammatory cytokine production by carbon monoxide involves the JNK pathway and AP-1. J Biol Chem 278: 36993-36998, 2003.

40. Clark JE, Naughton P, Shurey S, Green CJ, Johnson TR, Mann BE, Foresti R and Motterlini R: Cardioprotective actions by a water-soluble carbon monoxide-releasing molecule. Circ Res 93: e2-e8, 2003.

41. Kapitulnik J: Bilirubin: An endogenous product of heme degradation with both cytotoxic and cytoprotective properties. Mol Pharmacol 66: 773-779, 2004. 